\title{
Destruction of Scatter-wave Jamming Suppression for SAR Dual-channel Cancellation with Multi-jammers Mingxing $\mathrm{FANG}^{1, \mathrm{a}}$, Daping $\mathrm{BI}^{2, \mathrm{~b}}$, Aiguo $\mathrm{SHEN}^{3, \mathrm{c}}$, Yang ZHOU ${ }^{4, \mathrm{~d}}$ \\ ${ }^{1}$ Electronic Engineering Institute, Hefei, 230037, China \\ ${ }^{2}$ Electronic Engineering Institute, Hefei, 230037, China \\ ${ }^{3}$ Electronic Engineering Institute, Hefei, 230037, China \\ ${ }^{4}$ Electronic Engineering Institute, Hefei, 230037, China \\ aemail: mingxingfang89@163.com, bemail: DAPEEI@163.com \\ cemail: shenaiguo_405@sina.com, demail:zhouyang93@163.com
}

\section{Keywords: Synthetic Aperture Radar (SAR); Dual-channel Cancellation; Multi-jammers;} Scatter-wave Jamming

\begin{abstract}
SAR dual-channel cancellation technique can suppress the scatter-wave jamming of single jammer, so the scatter-wave jamming method of multi-jammers for SAR dual-channel cancellation system is proposed. First, the scatter-wave jamming suppression principle of dual-channel cancellation is reviewed. Then, the jamming model of multi-jammers for SAR dual-channel cancellation system is proposed, and the requirement of the interference phase and jammer configuration scheme are discussed. The method can also popularize to SAR multi-channel cancellation system, so it provides effective jamming way for multi-channel SAR. Theoretical analysis and computer simulation justify the validity and efficiency.
\end{abstract}

\section{Introduction}

Synthetic Aperture Radar(SAR) is an all-day and all-weather high-resolution senor that has been widely used in military investigating, mapping and matching to the end of guided missile ${ }^{[1]}$. Jamming technology against SAR has been a hotspot problem ${ }^{[2]}$ due to the huge influence that SAR plays on high-tech partial war. With the rapid development of SAR, single-channel SAR is hard to satisfy the demand, so multi-channel SAR is coming out ${ }^{[3]}$. Multi-channel SAR is better than single channel SAR in anti-interference. In general, suppression and cancelling, GMTI, phase interference, air region filtering are used for Multi-channel $\mathrm{SAR}^{[4-5]}$. At present, SAR multi-channel cancellation technology has become an important anti-interference measure. In [6], SAR dual-channel cancellation theory for direct-wave jamming is proposed. In [7], SAR tri-channel cancellation is used to suppress the blanket jamming and its loss of image information is less than dual-channel cancellation. A method of suppressing SAR scattering wave interference by dual-channel cancellation is proposed in [8]. To address the estimation problem of phase difference of interference signal in [8], an auto searching algorithm is proposed in [9] based on the principle of minimum power of scatter-wave jamming signal after cancellation. Since the development of single channel or multiple channels cancellation method of SAR greatly reduce the jamming performance of conventional methods, a method of multiple jammers is adopted to destroy the SAR dual-channel cancellation system in [10]. In [11], a rotating jammer is used to disable the InSAR two channels cancellation system. In this paper, we will use multi-jammers to destroy SAR dual-channel cancellation system for scatter-wave jamming.

\section{Scatter-wave Jamming Theory}

SAR scatter-wave jamming theory diagram is shown in Figure 1. When radar beam irradiation to the jammer, the signal is projected onto the selected scattering area after signal amplification. When SAR receives scatter-wave jamming signal, it will form multi-dimensional jamming performance 
from time domain and frequency domain, the polarization domain.

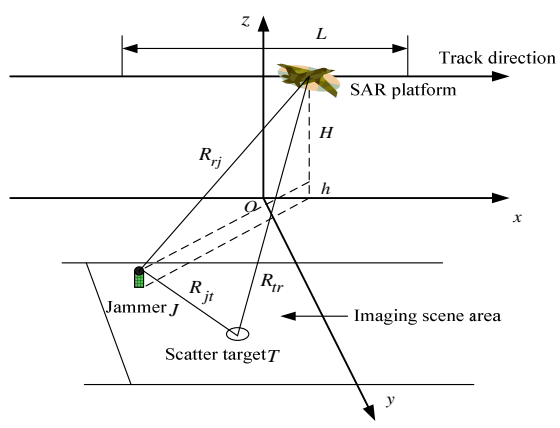

Fig.1. Scatter-wave jamming theory diagram

Assume that SAR platform flies along $x$ axis at speed $v$, its instantaneous coordinate is $\left(v t_{a}, 0, H\right)$. The coordinate of the jammer is $\left(x_{j}, y_{j}, h\right)$, The target coordinate in scatter area is $\left(x_{t}, y_{t}, 0\right)$. The spread path of jamming signal can be written as

$R_{j}\left(t_{a}\right)=R_{r j}\left(t_{a}\right)+R_{j t}+R_{t r}\left(t_{a}\right)$

where $R_{j t}=\sqrt{\left(x_{j}-x_{t}\right)^{2}+\left(y_{j}-y_{t}\right)^{2}+h^{2}}$ is the fixed distance of the jammer to the target, $R_{r j}\left(t_{a}\right)$ is the instantaneous distance of the jammer to the SAR platform, $R_{t r}$ is the instantaneous distance of the target to the SAR platform. Their Fresnel approximation can be represented as

$$
\begin{aligned}
& R_{r j}\left(t_{a}\right)=\sqrt{\left(v t_{a}-x_{j}\right)^{2}+y_{j}^{2}+(H-h)^{2}} \approx R_{j 0}-\frac{v x_{j}}{R_{j 0}} t_{a}+\frac{v^{2}}{2 R_{j 0}} t_{a}^{2} \\
& R_{t r}\left(t_{a}\right)=\sqrt{\left(v t_{a}-x_{0}\right)^{2}+y_{0}^{2}+H^{2}} \approx R_{t 0}-\frac{v x_{0}}{R_{t 0}} t_{a}+\frac{v^{2}}{2 R_{t 0}} t_{a}^{2}
\end{aligned}
$$

where $R_{j 0}$ is the initial distance of $R_{r j}\left(t_{a}\right), R_{t 0}$ is the initial distance of $R_{t r}\left(t_{a}\right)$. The linear frequency modulation signal of SAR can be expressed as

$$
s\left(t_{r}, t_{a}\right)=\operatorname{rect}\left(t_{r} / T_{p}\right) \exp \left[\mathrm{j} 2 \pi\left(f_{0} t+u_{r} t_{r}^{2} / 2\right)\right]
$$

where rect $(g)$ is rectangular window function, $t_{r}$ is the fast time in range, $t_{a}$ is the slow time in azimuth, $t=t_{r}+t_{a}$ is the whole time; $T_{p}$ is the pulse width, $f_{0}$ is the carrier frequency, $u_{r}$ is the frequency modulation slope. Ignoring the time-delay of the jammer, the fundamental frequency scatter-wave jamming signal which SAR receives can be written as

$$
s_{j}\left(t_{r}, t_{a}\right)=\sigma \operatorname{grect}\left(\frac{t_{r}-R_{j}\left(t_{a}\right) / c}{T_{p}}\right) \operatorname{rect}\left(\frac{t_{a}}{T_{L}}\right) \exp \left[\mathrm{j} \pi u_{r}\left(t_{r}-R_{j}\left(t_{a}\right) / c\right)^{2}\right] \exp \left[-\mathrm{j} 2 \pi R_{j}\left(t_{a}\right) / \lambda\right]
$$

where $\sigma$ is the target backscatter coefficient, $c$ is the speed of light, $T_{L}$ is the synthetic aperture time. The doppler center frequency and doppler frequency modulation slope are as follows:

$$
\left\{\begin{array}{l}
f_{c 1}=-\left.\frac{1}{\lambda} \mathrm{g} \frac{d R_{j}\left(t_{a}\right)}{d t_{a}}\right|_{t_{a}=0}=\frac{v x_{j}}{\lambda R_{j 0}}+\frac{v x_{t}}{\lambda R_{t 0}} \\
u_{a 1}=-\frac{1}{\lambda} \mathrm{g} \frac{d^{2} R_{j}\left(t_{a}\right)}{d t_{a}^{2}}=-\frac{v^{2}}{\lambda R_{j 0}}-\frac{v^{2}}{\lambda R_{t 0}}
\end{array}\right.
$$

The doppler center frequency and doppler frequency modulation slope of SAR matched filter are respectively $f_{c}=2 v x_{t} / \lambda R_{t 0}$ and $u_{a}=-2 v^{2} / \lambda R_{t 0}$.Compared with formula(6), the doppler parameters of jamming signal don't match with SAR matched filter because of $R_{j 0} \neq R_{t 0}$. The mismatch of doppler center frequency leads to the location deviation, and the mismatch of doppler frequency modulation slope leads to imaing defocusing in azimuth.

SAR matched filtering function in range is $h_{r}\left(t_{r}\right)=\operatorname{rect}\left(t_{r} / T_{p}\right) \exp \left(-\mathrm{j} \pi u_{r} t_{r}^{2}\right)$, so the matched 
filtering envelope result after range migration correction can be expressed as

$$
y_{j r}\left(t_{r}, t_{a}\right)=\sigma g\left(1-\frac{\left|t_{r}^{*}\right|}{T_{p}}\right) \operatorname{gsinc}\left(\pi u_{r} t_{r}^{*}\left(T_{p}-\left|t_{r}^{*}\right|\right)\right) \operatorname{gexp}\left[-\mathrm{j} 2 \pi R_{j}\left(t_{a}\right) / \lambda\right]
$$

where $t_{r}^{*}=t_{r}-\left(R_{j 0}+R_{t 0}+R_{j t}\right) / c$ is the fast time of echo arrival after range migration correction which is not related to the slow time. The peak time of jamming signal after compression is $t_{r}=\left(R_{j 0}+R_{t 0}+R_{j t}\right) / c$, so the jamming target image lags after the real image in range.

SAR matched filter function in azimuth is $h_{a}\left(t_{a}\right)=\operatorname{rect}\left(t_{a} / T_{L}\right) \exp \left(-\mathrm{j} \pi u_{a} t_{a}^{2}\right)$, so the matched filtering expressions of time domain in azimuth can be written as

$$
\begin{aligned}
y_{j a}\left(t_{r}, t_{a}\right) & =y_{j r}\left(t_{r}, t_{a}\right) \otimes h_{a}\left(t_{a}\right) \\
& =\int_{-\infty}^{+\infty} y_{j r}\left(t_{r}, \tau\right) h_{a}\left(t_{a}-\tau\right) d \tau
\end{aligned}
$$

Under the condition of Fresnel approximation, the SAR slow time signal in azimuth can still be seen as linear frequency modulation signal, so the formula (8) can be solved by principle of stationary phase. The integrating phase of slow time in azimuth can be written as

$$
\begin{aligned}
\phi(\tau) & =-2 \pi R_{j}(\tau) / \lambda-\pi u_{a}\left(t_{a}-\tau\right)^{2} \\
& =2 \pi f_{c 1} \tau+\pi u_{a 1} \tau^{2}-\pi u_{a}\left(t_{a}-\tau\right)^{2}
\end{aligned}
$$

Assume that $\left.\phi^{\prime}(\tau)\right|_{\tau=\tau^{*}}=0$, so the azimuth slow time $t_{a m}$ of stationary phase can be expressed as

$$
t_{a m}=\frac{x_{t}}{v}+\frac{x_{j} R_{t 0}-x_{t} R_{j 0}}{2 v R_{j 0}}+\frac{u_{a}-u_{a 1}}{u_{a}} \tau^{*}
$$

The output envelope after azimuth compression can be expressed as

$$
\left.y_{j a}\left(t_{r}, t_{a}\right)=\left(1-\frac{\left|t_{r}^{*}\right|}{T_{p}}\right) \oint 1-\frac{\left|t_{a}^{*}\right|}{T_{L}}\right) \operatorname{gsinc}\left(\pi u_{r} t_{r}^{*}\left(T_{p}-\left|t_{r}^{*}\right|\right)\right) \operatorname{gsinc}\left[\pi u_{a} t_{a}^{*}\left(T_{L}-\left|t_{a}^{*}\right|\right)\right]
$$

where $t_{a}^{*}=t_{a}-t_{a m}$ indicates the peak time, the constant term of $t_{a m}$ about $\tau^{*}$ about $t_{a}=t_{a m}$ denotes the azimuth peak position $x_{t}+\left(x_{j} R_{t 0}-x_{t} R_{j 0}\right) / 2 R_{j 0}$; the monomial term of $t_{a m}$ about $\tau^{*}$ denotes that the azimuth peak time changes with linear slope, it will leads to homogeneous broadening of peak position.

\section{Theory of Scatter-wave Jamming Suppression with SAR Dual-channel Cancellation}

SAR dual-channel cancellation theory diagram is shown in Figure 2. A, B two channel aperture antennas are arranged along the track direction, A antenna transmits radar signal, and B antenna receives signal. The antenna spacing meets dual-channel cancellation conditions:

$$
D=P R T g
$$

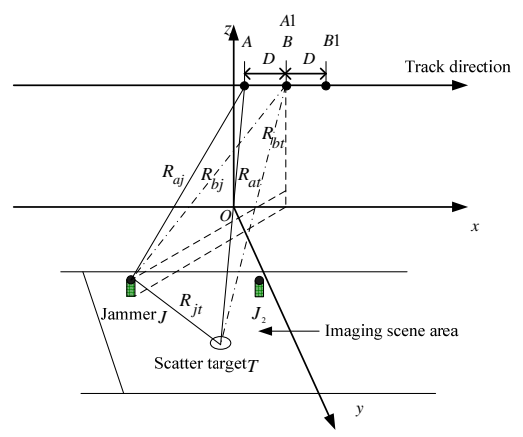

Fig.2. SAR dual-channel cancellation theory diagram

Assume $A$ and $B$ two antennas in Figure 2 are located in $n$ sampling point location, $A 1$ and $B 1$ two antennas are located in $n+1$ sampling point location. The received signals of the two antennas in $n$ 
sampling point location can be represented as

$$
\left\{\begin{array}{l}
s_{a}(n)=s_{a t}(n)+s_{a j}(n) \\
s_{b}(n)=s_{b t}(n)+s_{b j}(n)
\end{array}\right.
$$

where $s_{a t}(n)$ is the received real target signal of $A$ antenna in $n$ sampling point location, $s_{a j}(n)$ is the received scatter-wave jamming signal of $A$ antenna; $s_{b t}(n)$ is the received real target signal of $B$ antenna in $n$ sampling point location, $s_{b j}(n)$ is the received scatter-wave jamming signal of $B$ antenna. The location of $A$ antenna in $n+1$ sampling point location is the same with the location of $B$ antenna in $n$ sampling point location, so the received signal of $A$ antenna in $n+1$ sampling point location can be written as

$$
s_{a}(n+1)=s_{a t}(n+1)+s_{a j}(n+1)
$$

According to the signal transmission path, the received signal phase relationship between $B$ antenna in $n$ sampling point location and $A$ antenna in $n+1$ sampling point location can be expressed as

$$
\left\{\begin{array}{l}
\varphi_{b t}(n)=2 \pi\left(R_{a t}+R_{b t}\right) / \lambda \\
\varphi_{b j}(n)=2 \pi\left(R_{a j}+R_{j t}+R_{b t}\right) / \lambda \\
\varphi_{a t}(n+1)=2 \pi\left(2 R_{b t}\right) / \lambda \\
\varphi_{a j}(n+1)=2 \pi\left(R_{b j}+R_{j t}+R_{b t}\right) / \lambda
\end{array}\right.
$$

where $R_{a t}$ is the instantaneous slant distance of the scatter target $T$ to $A$ antenna, $R_{b t}$ is the instantaneous slant distance of the scatter target $T$ to $B$ antenna; $R_{a j}$ is the instantaneous slant distance of the jammer to $A$ antenna, $R_{b j}$ is the instantaneous slant distance of the jammer to $B$ antenna. According to (15), the received signal phase relationship between $B$ antenna in $n$ sampling point location and $A$ antenna in $n+1$ sampling point location can be expressed as

$$
\left\{\begin{array}{l}
\varphi_{b t}(n)=\varphi_{a t}(n+1)+\Delta \varphi_{t} \\
\varphi_{b j}(n)=\varphi_{a j}(n+1)+\Delta \varphi_{j}
\end{array}\right.
$$

where

$$
\left\{\begin{array}{l}
\Delta \varphi_{t}=2 \pi\left(R_{a t}-R_{b t}\right) / \lambda \\
\Delta \varphi_{j}=2 \pi\left(R_{a j}-R_{b j}\right) / \lambda
\end{array}\right.
$$

The received signal relationship formula between $B$ antenna in $n$ sampling point location and $A$ antenna in $n+1$ sampling point location can be expressed as

$$
\left\{\begin{array}{l}
s_{b t}(n)=s_{a t}(n+1) \operatorname{gexp}\left(\mathrm{j} \Delta \varphi_{t}\right) \\
s_{b j}(n)=s_{a j}(n+1) \operatorname{gexp}\left(\mathrm{j} \Delta \varphi_{j}\right)
\end{array}\right.
$$

where $\Delta \varphi_{j}$ is the required compensation phase of dual-channel cancellation. Due to difficult to accurately measure the distance value of SAR to the jammer, so it is difficult to calculate $\Delta \varphi_{j}$ of each sampling time phase by. In practice, we often use automatic phase searching algorithm to calculate $\Delta \varphi_{j}$ based on minimum energy of scatter-wave jamming signal after cancellation ${ }^{[9]}$. After $\Delta \varphi_{j}$ is known, we can use (19) to eliminate scatter-wave jamming signal.

$$
\begin{aligned}
\Delta s & =s_{b}(n)-s_{a}(n+1) \operatorname{gexp}\left(\mathrm{j} \Delta \varphi_{j}\right) \\
& =s_{a t}(n+1) \mathrm{g}\left(\exp \left(\mathrm{j} \Delta \varphi_{t}\right)-\exp \left(\mathrm{j} \Delta \varphi_{j}\right)\right) \\
& =s_{b t}(n) \mathrm{g}(1-\exp (\mathrm{j} \Delta \varphi))
\end{aligned}
$$

where $\Delta \varphi=\Delta \varphi_{j}-\Delta \varphi_{t}$. Substituting (17) into (19), it yields 


$$
\begin{aligned}
\Delta \varphi & =2 \pi\left(R_{a t}-R_{b t}+R_{b j}-R_{a j}\right) / \lambda \\
& \approx 2 \pi D\left(x_{t}-x_{j}\right) /\left(\lambda R_{r j 0}\right)
\end{aligned}
$$

where $R_{r j 0}$ is the shortest slant distance of SAR to the jammer. Combination (19) and (20), the scatter-wave jamming signal is eliminated after dual-channel cancellation, but the real target signal is modulated by $(1-\exp (\mathrm{j} \Delta \varphi))$ after cancellation. When $\Delta \varphi \approx 2 k \pi, k \in Z$, real target signal is close to zero, the imaging area dims. In special case, when $x_{t}=x_{j}$, we can get the $\Delta \varphi=0$, so the real target signal is zero; When $\Delta \varphi \approx(2 k+1) \pi$, the target signal gets stronger, the imaging area lights. Through the above analysis, the image produces light and shade area because of the dual-channel cancellation.

\section{Destruction of Scatter-wave Jamming Suppression for SAR Dual-channel Cancellation with Multi-jammers}

SAR dual-channel cancellation technique can suppress the scatter-wave jamming of single jammer, so the scatter-wave jamming method of multi-jammers for SAR dual-channel cancellation system is proposed. In order to simplify the model and analysis, the paper mainly studies about the destruction for SAR dual-channel cancellation based on two jammers. As shown in Figure 2, two jammers $\left(J_{1}\right.$ and $\left.J_{2}\right)$ carry out scatter-wave jamming for SAR at the same time. Assume that $\Delta \varphi_{j 1}$ is the Phase difference of scatter-wave jamming signal $J_{1}, \Delta \varphi_{j 2}$ is the phase difference of scatter-wave jamming signal from $J_{2}$. Thus

$$
\left\{\begin{array}{l}
s_{j 1 b}(n)=s_{j 1 a}(n+1) \operatorname{gexp}\left(\mathrm{j} \Delta \varphi_{j 1}\right) \\
s_{j 2 b}(n)=s_{j 2 a}(n+1) \operatorname{gexp}\left(\mathrm{j} \Delta \varphi_{j 2}\right)
\end{array}\right.
$$

where $s_{j 1 b}(n)$ is the jamming signal of $B$ antenna in $n$ sampling point location from $J_{1}, s_{j 1 a}(n+1)$ is the jamming signal of $A$ antenna in $n+1$ sampling point location from $J_{1} ; s_{j 2 b}(n)$ is the jamming signal of $B$ antenna in $n$ sampling point location from $J_{2}, s_{j 2 a}(n+1)$ is the jamming signal of $A$ antenna in $n+1$ sampling point location from $J_{2}$. Thus the jamming signals of two antennas from two jammers can be expressed as

$$
\left\{\begin{array}{l}
s_{a}(n+1)=s_{j 1 a}(n+1)+s_{j 2 a}(n+1) \\
s_{b}(n)=s_{j 1 b}(n)+s_{j 2 b}(n)
\end{array}\right.
$$

Substituting (22) into (21), it yields

$$
s_{b}(n)=s_{a}(n+1) \exp \left(\mathrm{j} \Delta \varphi_{j 1}\right)+s_{j 2 b}(n) g\left(1-\exp \left[\mathrm{j}\left(\Delta \varphi_{j 1}-\Delta \varphi_{j 2}\right)\right]\right)
$$

From (23), the jamming signal of $B$ antenna contains two parts, the first part is the result after phase compensation for $A$ antenna, so the jamming signal will be eliminated with single jammer. The second part can be considered as additional item generated by the two jammers, and it is the key to destroy the SAR two-channel cancellation system. The phase of second part is $\exp \left[\mathrm{j}\left(\Delta \varphi_{j 1}-\Delta \varphi_{j 2}\right)\right]$, when $\Delta \varphi_{j 1}-\Delta \varphi_{j 2}=2 k \pi$, the second part is zero. At this time, the phase difference between $B$ antenna in $n$ sampling point location and $A$ antenna in $n+1$ sampling point location is $\exp \left(\mathrm{j} \Delta \varphi_{j 1}\right)$, so the jamming signal can be also removed. Thus, in order to make the SAR dual-channel cancellation disable, we should make sure $\Delta \varphi_{j 1}-\Delta \varphi_{j 2} \neq 2 k \pi$.

From above analysis and discussion, we need to meet the condition of $\Delta \varphi_{j 1}-\Delta \varphi_{j 2} \neq 2 k \pi$. From (17), $\Delta \varphi_{j 1}=2 \pi\left(R_{a t 1}-R_{b t 1}\right) /\left(\lambda R_{r j 1}\right), \Delta \varphi_{j 2}=2 \pi\left(R_{a t 2}-R_{b t 2}\right) /\left(\lambda R_{r j 2}\right)$.To ensure the jamming real-time and effectiveness and increase the utilization efficiency of jamming power, jammer are usually arranged in close distance scattering area position. Assume the two jammers has same range 
position and different azimuth position, and the shortest slant distance from two jammers to SAR is $R_{r j 1} \approx R_{r j 2}=R_{r j 0}$.Thus

$$
\Delta \varphi_{j 1}-\Delta \varphi_{j 2} \approx 2 \pi D\left(x_{j 1}-x_{j 2}\right) /\left(\lambda R_{r j 0}\right)
$$

where $x_{j 1}-x_{j 2}$ is distance difference of the two jammers in Azimuth, so $\Delta \varphi_{j 1}-\Delta \varphi_{j 2}$ has a linear change with $x_{j 1}-x_{j 2}$. The change curve of phase difference with distance difference in Azimuth is shown in Figure 3. From Figure 3, the conditions of $\Delta \varphi_{j 1}-\Delta \varphi_{j 2} \neq 2 k \pi$ can be satisfied in general. in order to achieve a wide area of persistent jamming effect, we can arrange more than two jammers in different azimuth position.

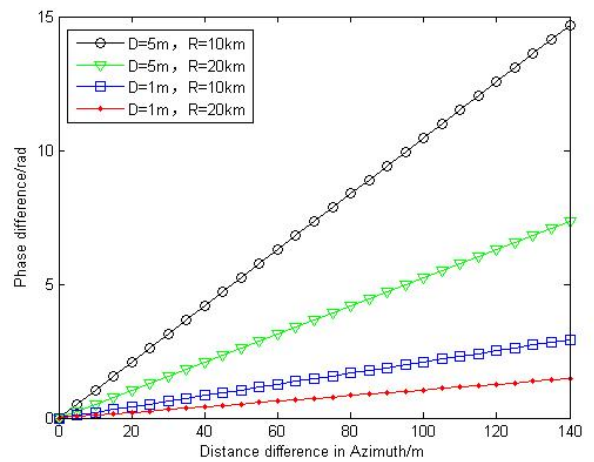

Fig.3. The change curve of phase difference with distance difference in Azimuth

\section{Test results}

The simulation parameters of SAR dual-channel cancellation system are shown in table I. The center coordinates of imaging scene is $[10000,0]$ (the unit is $\mathrm{m}$ ).

Table I. The simulation parameters

\begin{tabular}{cccc}
\hline Signal carrier frequency (GHz) & 10 & Channel antenna aperture (m) & 1 \\
Signal pulse width (us) & 20 & Synthetic aperture length (m) & 300 \\
Signal bandwidth (MHz) & 100 & Platform velocity (m/s) & 200 \\
Pulse repetition frequency (Hz) & 480 & Platform height (m) & 6000 \\
\hline
\end{tabular}

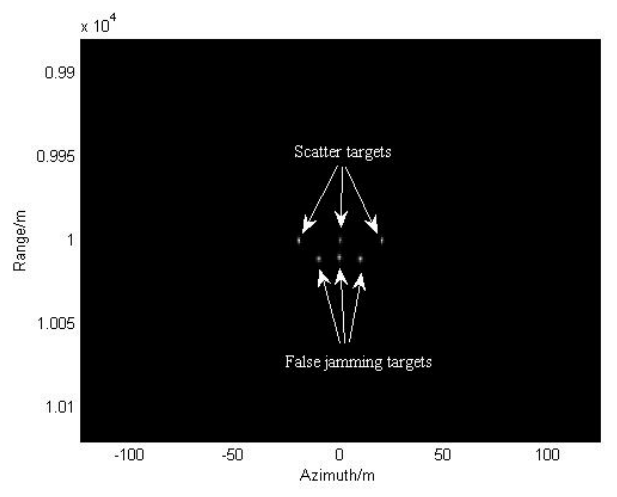

(a)Scatter-wave jamming imaging of single channel

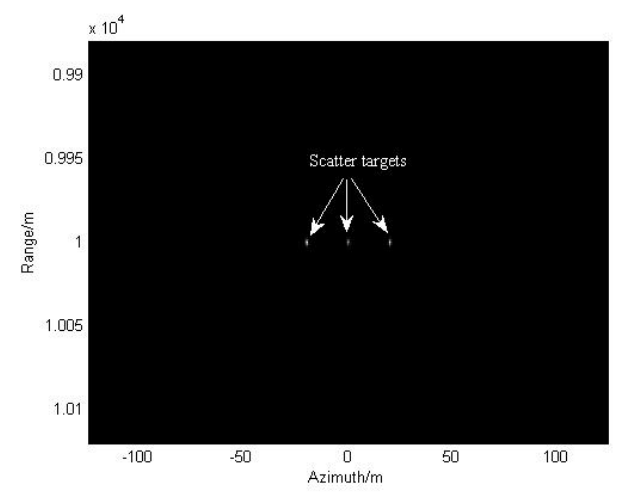

(b)Dual-channel cancellation jamming imaging

Fig.4. Scatter-wave jamming imaging of point targets before and after Dual-channel cancellation

According to the principle of scattering wave jamming and SAR dual-channel cancellation, first, verify point targets imaging results of scatter-wave jamming before and after dual-channel cancellation imaging results. Assuming the imaging area has three strong scattering targets, and the corresponding coordinates are $[10000,0],[10000, \pm 20]$. The coordinate of jammer is $[9800,0]$, and its height is $h=50 \mathrm{~m}$, and the jamming to signal ratio (JSR) is $10 \mathrm{~dB}$. Scatter-wave jamming imaging result is shown in Figure 4(a).Ignoring the tiny mismatch of frequency modulation slope, 
scatter-wave jamming can produce false targets for SAR, at this time the false targets lag behind the real targets about $10 \mathrm{~m}$ in range, and their corresponding coordinates in azimuth are $10 \mathrm{~m}, 0 \mathrm{~m}, 10 \mathrm{~m}$. Calculate the compensation phase $\Delta \varphi_{j}$ by formula (19) and suppress the scatter-wave jamming with dual-channel cancellation technique, so jamming imaging result after cancellation is shown in Figure 4 (b). From Figure 4 (b), we can see that all jamming targets are suppressed.

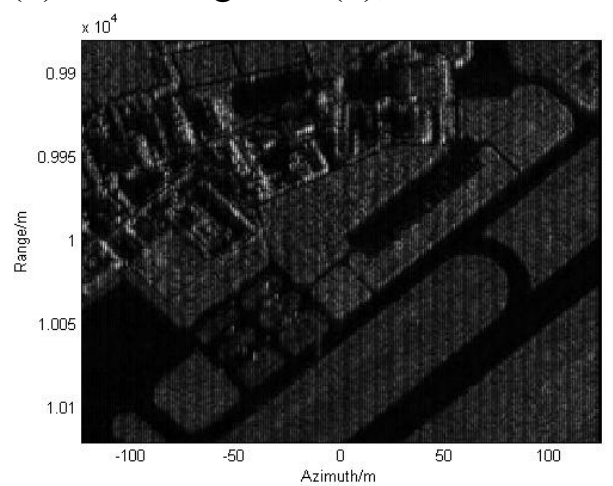

(a)Scene imaging without jamming

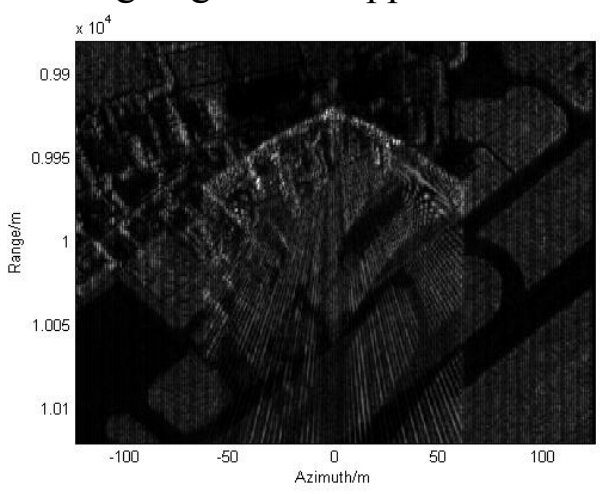

(b)Scatter-wave jamming scene imaging of single channel

Fig.5. Scatter-wave jamming scene imaging of single channel

In the same simulation conditions, verify scene imaging results of scatter-wave jamming before and after dual-channel cancellation imaging results. The scene imaging without jamming is shown in Figure 5 (a), and the scatter-wave jamming scene imaging of single channel is in Figure 5 (b), From them, we can see that scatter-wave jamming scene imaging can form superposition jamming effect on real imaging scene. According to the principle of scatter-wave jamming, the jamming scene has compression and torsion interference scene compared with the real scene, so it has mixed jamming effect of suppression and deception. Suppressing the scatter-wave jamming with dual-channel cancellation technique, the cancellation result is shown in Figure 6. From the comparison of Figure 5 and Figure 6, we can see that the jamming scene is removed. Because of the dual-channel cancellation, the image produces light and shade area in range, and the black stripe will cause the loss of real image information.

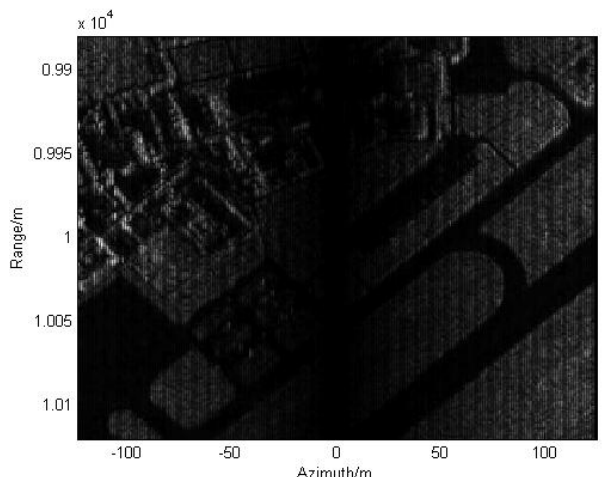

Fig.6. Dual-channel cancellation scene imaging of single jammer

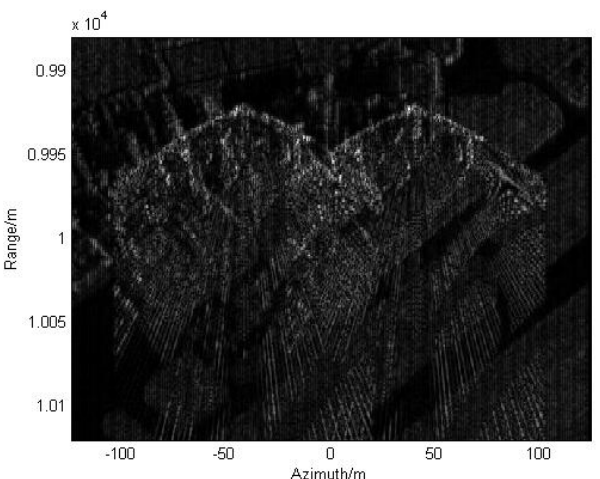

Fig.7. Dual-channel cancellation scene imaging of double jammers

From the above simulation analysis, although the SAR dual-channel cancellation will make the real image form light and shade change, and cause the loss of the real image information, but the SAR two-channel cancellation can effectively suppress the scatter-wave jamming of single jammer, so we must use more than one jammer to destroy SAR two-channel cancellation system. Assume that we have two jammers, and their corresponding coordinates are $[9800, \pm 50]$. At this time, we can only estimate the wrong compensation phase of $\Delta \hat{\varphi}_{j}$, and the last cancellation result is shown in Figure 7. We can see that the scatter-wave jamming still exist in the real image, so SAR two-channel cancellation system is disabled. 


\section{Conclusion}

SAR dual-channel cancellation technique can suppress the scatter-wave jamming of single jammer. Multi-jammers can make the SAR dual-channel cancellation system cannot estimate compensation phase of every slow time, and ensure the scattering wave jamming effect for SAR dual-channel cancellation. This method can be extended to multiple jammers damage SAR multi-channel cancellation for scatter-wave jamming, and effectively improve the jamming capacity for multi-channel SAR.

\section{References}

[1] Cumming I G, Wong F H. Digital processing of synthetic aperture radar data: algorithms and implementation [M]. Boston: Artech House, 2009.

[2] Jia Xin, Ye Wei, Wu Yan-hong, et al. Electronic countermeasure techmology to synthetic aperture radar[M]. Beijng: National Defense Industry Press, 2014.

[3] Sjogren T K, Viet T V, Pettersson M I, et al. Suppression of clutter in multichannel SAR-GMTI[J]. IEEE Transactions on Geoscience and Remote Sensing, 2014, 52(7):4005-4013.

[4] L Rosenberg, D.Gray. Anti-jamming techniques for multichannel SAR imaging[C]. IEE Proc-Radar Sonar Navig, 2006, 153(3):234-242.

[5] Huang Long, Dong Chun-xi, Shen Zhi-bo, et al. The influence of rebound jamming on SAR-GMTI[J]. IEEE Geoscience and Remote Sensing Letters, 2015, 12(2):399-403.

[6] Wang Ai-jun, Chu Jian-xiang, Wang Ping, et al. Affection of two-channel cancellation method suppressing SAR jamming to target[J]. Aerospace Electronic Warfare, 2007, 23(1):35-39.

[7] Ma Xiao-yan, Qin Jiang-min, He Zhao-hui, et al. Three-channel cancellation of SAR Blanketing jamming suppression[J]. Acta Electronica Sinica, 2007, 35(6):1015-1020

[8] Gan Rong-bing, Wang Jian-guo, He Chuan. Rebound jamming suppression by two-channel SAR[J]. Signal Processing, 2005, 21(1):27-30.

[9] Gan Rong-bing, Wang Jian-guo, He Chuan. Phase estimation in rebound jamming suppression by two-channel SAR[J]. Acta Electronica Sinica, 2005, 33(9):1691-1693.

[10] Huang Long, Dong Chun-xi, Zhao Guo-qing. Investigation on countermeasure against SAR dual-channel cancellation technique with multi-jammers[J]. Journal of Electronics \& Information Technology, 2014, 34(4):903-907.

[11] Huang Long, Dong Chun-xi, Shen Zhi-bo. Investigation on countermeasure against InSAR dual-channel cancellation technique with Multi-antenna Jammer[J]. Journal of Electronics \& Information Technology, 2015, 37(4):913-918. 